\title{
A detailed look at chemical abundances in the Magellanic Clouds
}

\author{
Richard A. Shaw ${ }^{1}$, Ting-Hui Lee ${ }^{1,2}$, Letizia Stanghellini ${ }^{1}$, James E. \\ Davies $^{1}$, D. Anibal García-Hernández ${ }^{3,4}$, Pedro García-Lario ${ }^{5}$, \\ Jose-Vicente Perea-Calderón $^{6}$, Eva Villaver ${ }^{7}$, Arturo Manchado ${ }^{3,4,8}$, \\ Stacy Palen ${ }^{9}$, and Bruce Balick ${ }^{10}$ \\ ${ }^{1}$ National Optical Astronomy Observatory, Tucson, AZ 85719, USA \\ ${ }^{2}$ Dept. of Physics \& Astronomy, Western Kentucky Univ., Bowling Green, KY 42101, USA \\ ${ }^{3}$ Instituto de Astrofísica de Canarias, vía Láctea s/n, La Laguna, E-38200 Tenerife, Spain \\ ${ }^{4}$ Departamento de Astrofísica, Universidad de La Laguna, E-38201 Tenerife, Spain \\ ${ }^{5}$ Herschel Science Centre, European Space Astronomy Centre, E-28080 Madrid, Spain \\ ${ }^{6}$ European Space Astronomy Centre, INSA S. A., E28080, Madrid, Spain \\ ${ }^{7}$ Departamento de Física Teórica C-XI, Univ. Autónoma de Madrid, E-28049 Madrid, Spain \\ ${ }^{8}$ Consejo Superior de Investigaciones Cientficas, E-28006 Madrid, Spain \\ ${ }^{9}$ Physics Dept., Weber State University, Ogden, UT 84408, USA \\ ${ }^{10}$ Astronomy Dept., Univ. of Washington, Seattle, WA 98195, USA
}

\begin{abstract}
We determine elemental abundances of $\mathrm{He}, \mathrm{N}, \mathrm{O}, \mathrm{Ne}, \mathrm{S}$, and $\mathrm{Ar}$ in Magellanic Cloud planetary nebulae $(\mathrm{PNe})$ using direct methods and a large set of observed ions, minimizing the need for ionization correction factors. In contrast to prior studies, we find a clear separation between Type I and non-Type I PNe in these low-metallicity environments, and no evidence that the $\mathrm{O}-\mathrm{N}$ nucleosynthesis cycle is active in low-mass progenitors. We find that the $\mathrm{S} / \mathrm{O}$ abundance ratio is anomalously low compared to H II regions, confirming the "sulfur anomaly" found for Galactic PNe. We also found that $\mathrm{Ne} / \mathrm{O}$ is elevated in some cases, raising the possibility that Ne yields in low-mass AGB stars may be enhanced at low metallicity.
\end{abstract}

Keywords. planetary nebulae: general, ISM: abundances, galaxies: individual (LMC, SMC)

\section{Observations and Analysis}

We present an analysis of elemental abundances of $\mathrm{He}, \mathrm{N}, \mathrm{O}, \mathrm{Ne}, \mathrm{S}$, and Ar in Magellanic Cloud planetary nebulae (PNe). The abundances were derived from a combination of deep, high dispersion optical spectra, space-based mid-IR spectra, and space-based UV and optical spectra. Through the use of wider wavelength coverage, higher resolution, and more accurate flux calibration, we have been able to derive ionic abundances from collisionally excited emission lines which are the most accurate available for Magellanic Cloud PNe. We used the ionic abundances, including those from ionization stages not observable in the optical, to examine the accuracy of some commonly used recipes for ionization correction factors (ICFs). We find that most of our adopted ICFs (from Kingsburgh \& Barlow 1994; Kwitter \& Henry 2001) work very well even in the limit of substantially sub-Solar metallicities, except for PNe with very high ionization.

\section{Results}

Our abundance analysis shows enhancements of $\mathrm{He}$ and $\mathrm{N}$ that are predicted from prior dredge-up processes in the AGB progenitors, and which agree well with expectations from nebular morphology. Figure 1 shows a clear separation of Type I PNe from other types 

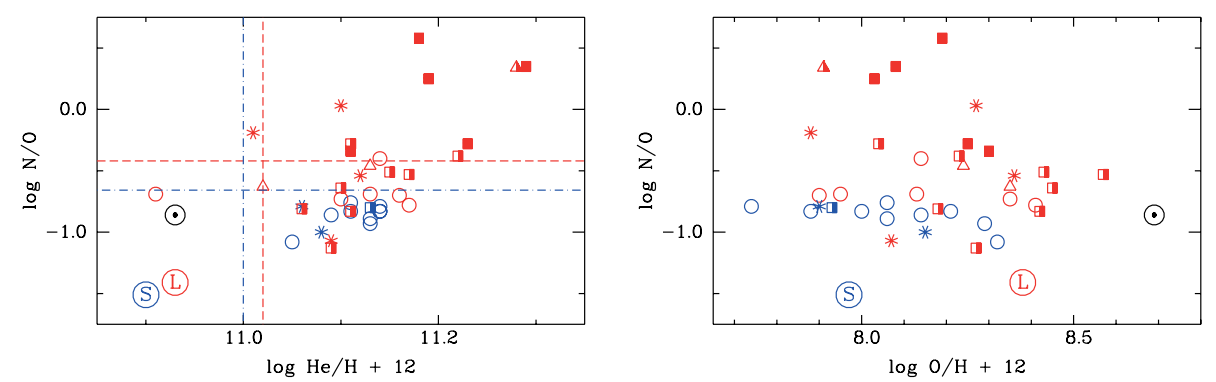

Figure 1. Abundance ratios $\mathrm{N} / \mathrm{O}$ vs. $\mathrm{He} / \mathrm{H}$ (left) and $\mathrm{O} / \mathrm{H}$ (right) for $\mathrm{PNe}$ in the Large (red symbols) and Small (blue) Magellanic Clouds. Type I PNe, including bipolars (filled squares) lie to the upper right of the dashed lines (Shaw et al. 2010). Averages for H II regions (Dennefeld 1989) are indicated (circled $L, S$ ), as are solar abundances (sun symbols) (Asplund et al. 2009).
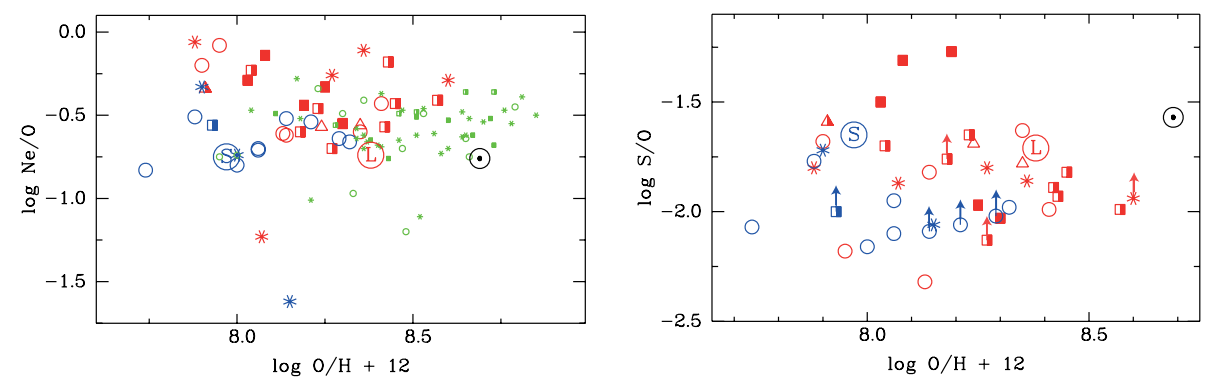

Figure 2. Abundances of Ne/O (left) and S/O (right) vs. O for Magellanic Cloud PNe. Symbols as in Fig. 1. Also plotted (left) are Ne/O ratios from the Galactic PN sample (small symbols) of Stanghellini et al. (2006).

(using the Magellanic Cloud thresholds discussed in Shaw, et al. 2010), and also that the O-N nucleosynthesis cycle does not appear to operate in low-mass progenitors.

The predicted yields from nucleosynthesis in the prior AGB phase for the alphaelements S and Ar are predicted to remain constant (Karakas 2010), and should therefore track the $\mathrm{O}$ abundance very well, while $\mathrm{Ne}$ may be enhanced in low-mass PN progenitors at low metallicity. As Fig. 2 shows, the $\mathrm{Ne} / \mathrm{O}$ vs. O relation shows significant dispersion, with many LMC PNe showing relatively high Ne abundance. The S abundance, where all ionization stages lower than $\mathrm{S}^{4+}$ are observed, is systematically low compared to H II regions in each galaxy by an amount that is consistent with the "sulfur anomaly" (Henry et al. 2004). These depressed S abundances cannot be simply attributed to observational error or faulty ICFs. Other possible explanations include faulty atomic data, and peculiar star formation histories that skew the ratios of the alpha elements relative to $\mathrm{O}$.

\section{References}

Asplund, M., Grevesse, N., Jacques Sauval, A., \& Scott, P. 2009, ARAA, 47, 481

Dennefeld, M. 1989, Recent Developments of Magellanic Cloud Research, ed. K. S. de Boer, F. Spite, E3 G. Stasinska (Paris: Obs. de Paris), 107

Henry, R. B. C., Kwitter, K. B., \& Balick, B. 2004, AJ, 127, 2284

Karakas, A. 2010, MNRAS, 403, 1413

Kingsburgh, R. L. \& Barlow, M. J. 1994, MNRAS, 271, 257 (KB94)

Kwitter, K. B. \& Henry, R. B. C. 2001, ApJ, 562, 804

Shaw, R. A., et al. 2010, ApJ, 717, 562

Stanghellini, L., et al. 2006, ApJ, 717, 562 